\title{
Endocannabinoid metabolism in neurodegenerative diseases
}

\author{
Chu Chen
}

Neuroscience Center of Excellence, School of Medicine, Louisiana State University Health Sciences Center, New Orleans, LA 70112, USA.

Correspondence to: Dr. Chu Chen, Neuroscience Center of Excellence, School of Medicine, Louisiana State University Health Sciences Center, 2020 Gravier Street, New Orleans, LA 70112, USA. E-mail: cchen@1suhsc.edu; chen502@gmail.com

How to cite this article: Chen C. Endocannabinoid metabolism in neurodegenerative diseases. Neuroimmunol Neuroinflammation 2016;3:268-70. Article history: Received: 14-11-2016 Accepted: 15-11-2016 Published: 15-12-2016

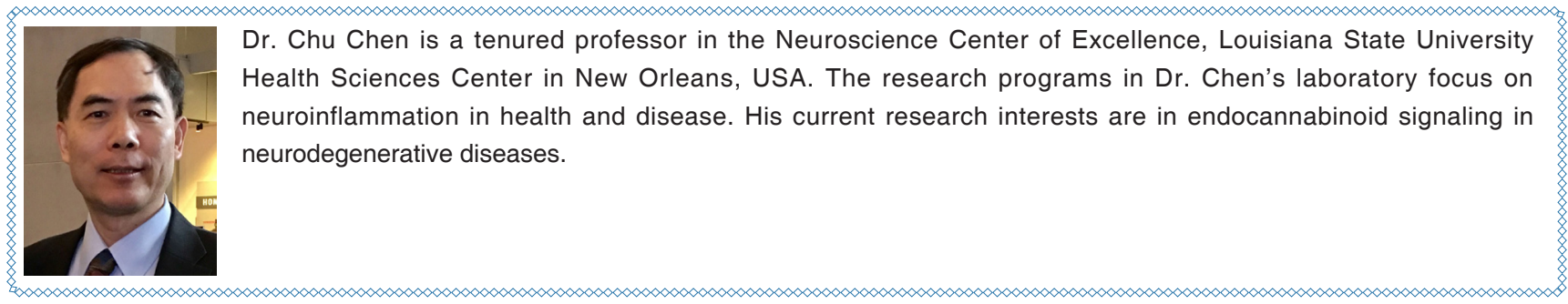

Endocannabinoids are endogenous lipid mediators contributing to a variety of physiological, pharmacological, and pathological processes primarily through acting on cannabinoid receptors (CB1R and CB2R), which are targets of $\Delta^{9}$-tetrahydrocannabinol $\left(\Delta^{9}-\mathrm{THC}\right)$, the major psychoactive ingredient in marijuana. ${ }^{[1]} \quad$ Although $\mathrm{N}$-arachidonoyl ethanolamide is the first identified endocannabinoid, 2-arachidonoylglycerol (2-AG), the second identified endocannabinoid, is the most abundant ligand produced in our body and a full agonist for CB1R and $\mathrm{CB} 2 \mathrm{R}^{\left[{ }^{[2]}\right.}$ It has been well recognized that 2-AG is a retrograde messenger modulating synaptic transmission and plasticity at both inhibitory GABAergic and excitatory glutamatergic synapses in the brain. ${ }^{[2-4]}$ In particular, augmentation of 2-AG signaling by inhibition of its metabolism has been attracted attention recently due to its profound anti- inflammatory and neuroprotective properties. ${ }^{[4,5]}$

2-AG is synthesized largely from diacylglycerol by diacylglycerol lipase $\alpha$ and $\beta$ and primarily hydrolyzed by the enzyme monoacylglycerol lipase (MAGL) to glycerol and arachidonic acid (AA), a precursor of prostaglandins and leukotrienes [Figure 1]. And $2-A G$ is also degraded by the enzymes $\alpha / \beta$ hydrolase domain-containing protein 6 and 12 and metabolized oxidatively by cyclooxygenase 2 (COX-2). Apparently, $2-A G$ is a very unstable bioactive lipid mediator, and it is easily and rapidly degraded by these enzymes upon its synthesis. ${ }^{[2]}$ It has been estimated that $85 \%$ of $2-A G$ is metabolized by MAGL in the brain. ${ }^{[6]}$ Arachidonic aciddelivered prostaglandins and leukotrienes are generally proinflammatory and neurotoxic, whereas $2-A G$ is capable of resolving neuroinflammation and protecting neurons from harmful insults. ${ }^{[4,5,7]}$ This suggests that

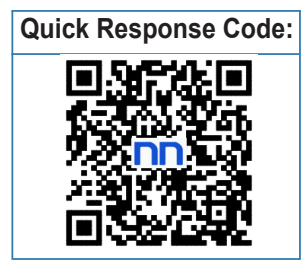


disruption of MAGL would be a promising strategy to enhance anti-inflammatory and neuroprotective 2-AG signaling, while reducing proinflammatory and neurotoxic eicosanoid (e.g. $\mathrm{PGE}_{2}$ ) levels [Figure 1].

Alzheimer's disease (AD) is the most common cause of dementia in elderly. While the etiology of $A D$ is multifactorial, accumulating evidence implicates traumatic brain injury (TBI) as an epigenetic risk factor in $A D$ development and dementia. Chronic traumatic encephalopathy (CTE) is the most recently defined TBI-caused neurodegenerative disease, and neuropathology and neurocognitive deficits in CTE are similar to those in AD. ${ }^{[4,8-10]}$ The significant similarities and overlap in the spectrum of changes in neuropathology, neurobiology, synaptic and neurocognitive deficits between CTE and AD suggest that CTE, in essence, is a TBI-triggered AD-like neurodegenerative disease. Repetitive or multiple brain injuries may lead to AD-like neuropathology, impairments in synaptic and cognitive functions, and dementia. ${ }^{[8-10]}$ However, there are currently no effective therapies to prevent and treat $A D$ and TBI-caused AD-

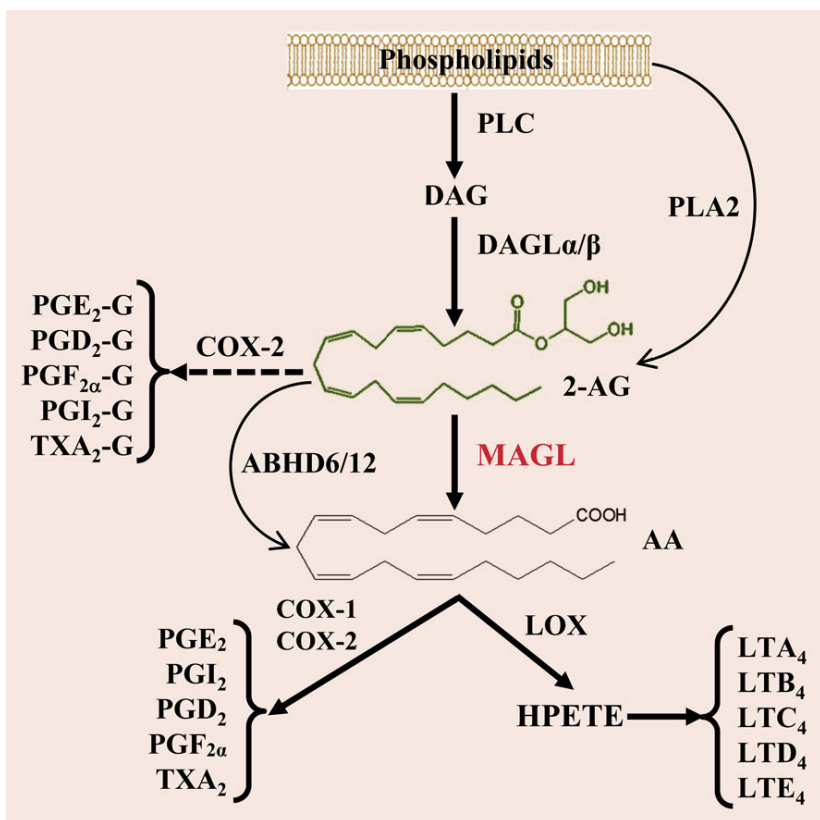

Figure 1: Pathways of 2-AG synthesis and metabolism. 2-AG is largely synthesized from DAG, which is formed from membrane phospholipids through PLC, by DAGL $\alpha / \beta$. 2-AG in the brain is primarily hydrolyzed by MAGL to glycerol and $A A$. It is also hydrolyzed by $A B H D 6 / 12$ to $A A$ and metabolized oxidatively by COX-2 to form a new type of prostaglandin glycerol esters when expression and activity of COX-2 are excessively elevated during inflammation. AA is a precursor of prostaglandins through the enzymes COX-1/2 and HPETE through the enzyme arachidonate 5-LOX to form leukotrienes (LTA4 to E4). 2-AG: 2-arachidonoylglycerol; PLC: phospholipase C; DAG: diacylglycerol; DAGL $\alpha / \beta$ : diacylglycerol lipase $\alpha$ and $\beta$; PLA2: phospholipase A2; AA: arachidonic acid; COX-2: cyclooxygenase-2; MAGL: monoacylglycerol lipase; ABHD6/12: $\alpha / \beta$ hydrolase domaincontaining protein 6 and 12; HPETE: hydroperoxyeicosatetraenoic acid; LOX: lipoxygenase like neurodegenerative disease or to halt progression of diseases. Earlier studies show that 2-AG protects neurons against brain trauma in a mouse model of closed head injury. ${ }^{[7]}$ Recent studies provide evidence that inactivation of MAGL reduces neuroinflammation, $A \beta$ accumulation and deposition, tau phosphorylation, and neurodegeneration and improves synaptic and neurocognitive functions in several animal models of neurodegenerative diseases, including $A D$, Parkinson's disease (PD), and TBI. ${ }^{[8,11-14]}$ This suggests that manipulations of 2-AG metabolism may provide novel pharmacotherapies for these intractable neurodegenerative diseases..$^{[4,8,11,12]}$

Although disruption of MAGL ameliorates neuropathology and prevent synaptic and cognitive declines in animal models of neurodegenerative diseases, ${ }^{[4,8,11-14]}$ the signaling pathways that mediate these beneficial effects produced by MAGL inhibition are still unclear. MAGL loss-of-function enhances anti-inflammatory and neuroprotective 2-AG signaling and decreases proinflammatory and neurotoxic prostaglandins and leukotrienes [Figure 1]. It is possible that the beneficial effects produced by MAGL inhibition are mediated either by enhanced 2-AG signaling through $\mathrm{CB} 1 \mathrm{R}$ or CB2R-depdendent mechanism or by reduced eicosanoid levels through cannabinoid receptor-independent mechanisms..$^{[4,8,11-14]}$ It has been proposed that peroxisome proliferator-activated receptor $\gamma$ (PPAR $\gamma$ ), a nuclear receptor that displays significant anti-inflammatory properties, is a target of endocannabinoids. ${ }^{[15,16]}$ Recent studies demonstrated that suppression of neuroinflammation and alleviation of $A D$ neuropathology by 2-AG or inactivation of MAGL are mediated via PPAR $\gamma,{ }^{[5,11,16]}$ suggesting that PPAR $\gamma$ is likely a downstream signaling molecule of 2-AG. Despite the fact that the mechanisms by which inhibition of 2-AG metabolism alleviates neuroinflammation and neuropathology and prevents deterioration in synaptic and cognitive functions in animal models of $A D, P D$, and TBI remain to be elucidated, MAGL is a promising therapeutic target for neurodegenerative diseases.

\section{Financial support and sponsorship}

This article is financed by National Institutes of Health grants (No. NS076815).

\section{Conflicts of interest}

There are no conflicts of interest.

\section{Patient consent}

There is no patient involved.

\section{Ethics approval}

This article does not contain any studies with human participants or animals. 


\section{REFERENCES}

1. Gaoni Y, Mechoulam R. Isolation, structure and partial synthesis of an active constituent of hashish. J Am Chem Soc 1964;86:1646-7.

2. Kano M, Ohno-Shosaku T, Hashimotodani Y, Uchigashima M, Watanabe M. Endocannabinoid-mediated control of synaptic transmission. Physiol Rev 2009;89:309-80.

3. CastilloPE, Younts TJ, ChávezAE, Hashimotodani Y.Endocannabinoid signaling and synaptic function. Neuron 2012;76:70-81.

4. $\mathrm{Xu} \mathrm{JY,} \mathrm{Chen} \mathrm{C}$. Endocannabinoids in synaptic plasticity and neuroprotection. Neuroscientist 2015;21:152-68.

5. Zhang J, Chen C. Endocannabinoid 2-arachidonoylglycerol protects neurons by limiting COX-2 elevation. J Biol Chem 2008;283:22601-11.

6. Blankman JL, Simon GM, Cravatt BF. A comprehensive profile of brain enzymes that hydrolyze the endocannabinoid 2-arachidonoylglycerol. Chem Biol 2007; 14:1347-56.

7. Panikashvili D, Simeonidou C, Ben-Shabat S, Hanus L, Breuer A, Mechoulam R, Shohami E. An endogenous cannabinoid (2-AG) is neuroprotective after brain injury. Nature 2001;413:527-31.

8. Zhang J, Teng $\mathrm{Z}$, Song $\mathrm{Y}$, $\mathrm{Hu} \mathrm{M}$, Chen $\mathrm{C}$. Inhibition of monoacylglycerol lipase prevents chronic traumatic encephalopathylike neuropathology in a mouse model of repetitive mild closed head injury. J Cereb Blood Flow Metab 2015;35:443-53.

9. Gupta R, Sen N. Traumatic brain injury: a risk factor for neurodegenerative diseases. Rev Neurosci 2016;27:93-100.

10. Djordjevic J, Sabbir MG, Albensi BC. Traumatic brain injury as a risk factor for Alzheimer's disease: is inflammatory signaling a key player? Curr Alzheimer Res 2016;13:730-8.

11. Zhang J, Hu M, Teng Z, Tang YP, Chen C. Synaptic and cognitive improvements by inhibition of 2-AG metabolism are through upregulation of microRNA-188-3p in a mouse model of Alzheimer's disease. J Neurosci 2014;34:14919-33.

12. Chen R, Zhang J, Wu Y, Wang D, Feng G, Tang YP, Teng Z, Chen C. Monoacylglycerol lipase is a therapeutic target for Alzheimer's disease. Cell Rep 2012;2:1329-39.

13. Nomura DK, Morrison BE, Blankman JL, Long JZ, Kinsey SG Marcondes MC, Ward AM, Hahn YK, Lichtman AH, Conti B, Cravatt BF. Endocannabinoid hydrolysis generates brain prostaglandins that promote neuroinflammation. Science 2011;334:809-13

14. Piro JR, Benjamin DI, Duerr JM, Pi Y, Gonzales C, Wood KM, Schwartz JW, Nomura DK, Samad TA. A dysregulated endocannabinoid-eicosanoid network supports pathogenesis in a mouse model of Alzheimer's disease. Cell Rep 2012;1:617-23.

15. O'Sullivan SE. Cannabinoids go nuclear: evidence for activation of peroxisome proliferator-activated receptors. $\mathrm{Br} J$ Pharmacol 2007; $152: 576-82$

16. Du H, Chen X, Zhang J, Chen C. Inhibition of COX-2 expression by endocannabinoid 2-arachidonoylglycerol is mediated via PPAR- $\gamma . \mathrm{Br}$ J Pharmacol 2011;163:1533-49. 